\title{
Prática de atividade física em quatro domínios em idosos com hipertensão arterial referida no Município de São Paulo: estudo de base populacional
}

\author{
Physical activity in four domains in elderly patients with self-reported \\ hypertension in São Paulo: a population-based study
}

\section{Kaio Henrique Correa Massa' Vanessa Valente Guimarães ${ }^{1}$ Chester Luiz Galvão César² Marilisa Berti de Azevedo Barros ${ }^{3}$ Luana Carandina ${ }^{4}$ Moisés Goldbaum ${ }^{5}$ Alex Antonio Florindo ${ }^{1}$}

1. Escola de Artes, Ciências e Humanidades, USP, São Paulo, SP, Brasil.

2. Departamento de Epidemiologia da Faculdade de Saúde Pública, USP, São Paulo, SP, Brasil.

3. Departamento de Saúde Coletiva, UNICAMP, Campinas, SP, Brasil.

4. Departamento de Saúde Pública,

Faculdade de Medicina de Botucatu, UNESP, Botucatu, SP, Brasil.

5. Departamento de Medicina Preventiva, Faculdade de Medicina, USP, São Paulo, SP, Brasil.

\section{ENDEREÇOPARA CORRESPONDÊNCIA}

Kaio Henrique Correa Massa

Rua Capela, 82

Pqe. Brasilia, Guarulhos, SP

07243-120

kaio.massa@hotmail.com

$\begin{array}{ll}\text { - Recebido: } & 07 / 06 / 2011 \\ \text { - Re-submissão: } & 14 / 08 / 2011 \\ \text { - Aceito: } & 05 / 09 / 2011\end{array}$

\section{Resumo}

A prática de atividade física (AF), no contexto de prevenção de doenças crônicas não-transmissíveis, é uma indicação de tratamento não-medicamentoso crescente, principalmente relacionada a patologias como a hipertensão arterial sistêmica (HAS), doença de alta prevalência populacional que caracteriza um dos principais fatores de risco para as doenças cardiovasculares e cerebrovasculares, maiores causas de mortalidade mundiais na modernidade. Avaliar a prática de AF em quatro domínios em população de 60 anos ou mais que referiu HAS segundo as características sociodemográficas. Este trabalho decorre do Inquérito de Saúde no Município de São Paulo, ISA-Capital 2003, estudo transversal de base populacional, com dados coletados por entrevista domiciliar. Investigou-se a prática de AF em quatro domínios através do IPAQ versão longa. Utilizou-se análise de Regressão de Poisson para modelos multivariados. A amostra do estudo totalizou 395 idosos, portadores autorreferidos de HAS Observou-se $6,2 \%$ de ativos na prática de AF no trabalho, 16,9\% na locomoção, 41,4\% na doméstica e $19,6 \%$ no lazer. A prática de AF no trabalho teve associação com sexo masculino e menor faixa etária; na locomoção não apresentou associação significativa com as variáveis estudadas; na doméstica observou-se associação com sexo feminino, menor faixa etária e situação conjugal de viuvez; e no lazer houve associação com maior escolaridade. Observou-se diferentes associações entre a prática recomendada de AF, nos quatro domínios, e variáveis sociodemográficas na população idosa com HAS autorreferida no Município de São Paulo. No geral, a prática de atividade física foi baixa devendo ser estimulada nessa população.

Palavras-chave: Atividade física; Hipertensão arterial; Idosos.

\section{Abstract}

The practice of physical activity (PA) in the context of prevention of chronic non-communicable diseases is an indication of increasing non-drug treatment, mainly related to diseases such as hypertension, a disease of high prevalence population featuring one of the main risk factors for cardiovascular and cerebrovascular diseases, actually the greatest cause of mortality in world. To estimate the practice of PA in four areas where the population aged 60 or older who reported hypertension according to sociodemographic characteristics. This work results from Health Survey in São Paulo, ISA-Capital 2003, population-based cross-sectional study, with data collected through residence interview. An investigation into the practice of PA in four areas through the long version of IPAQ. Poisson regression analysis has been used for miscellaneous models. The study sample add up to 395 old-aged, self-referred bearer of hypertension. There was $6.2 \%$ of assets in the practice of PA at work, $16.9 \%$ in transportation, $41.4 \%$ at home and $19.6 \%$ at leisure. The practice of PA at work was associated with males and lower age group; locomotion was not significantly associated with the variables studied, at home there was an association with female gender, lower age and marital status of widowhood, and at leisure was associated with higher scholarity. Observed associations between the different practice of PA in the four areas and sociodemographic variables in the elderly with self-referred hypertension in São Paulo. Overall, the practice of physical activity was low in this population and must be estimulated.

Keywords: Physical activity; Hypertension; Elderly. 


\section{INTRODUÇÃO}

Os novos paradigmas em promoção da saúde e prevenção de doenças priorizam, dentre outras estratégias, o desenvolvimento e manutenção de um estilo de vida ativo, considerando a prática acumulada de atividade física (AF) em seus quatro domínios: lazer, locomoção ou deslocamento, trabalho e doméstica. ${ }^{1,2}$ No campo da prevenção e tratamento de doenças crônicas, a prática de atividade física é indicada como comportamento preventivo associado à saúde, bem como tratamento não-medicamentoso de algumas doenças de alta prevalência, como hipertensão arterial (HA), diabetes e obesidade. ${ }^{2,3}$

A hipertensão arterial acomete aproximadamente $25,0 \%$ da população adulta mundial e é a doença crônica mais frequente na população idosa, com prevalências superiores a $50,0 \%$, crescentes com o decorrer da idade. ${ }^{4}$ No Brasil, a prevalência de $\mathrm{HA}$ referida está estimada em $21,6 \%$ na população adulta $^{5}$ e em $55,0 \%$ na população idosa, ${ }^{6}$ segundo dados do VIGITEL 2006, em investigação por inquérito telefônico de 26 capitais brasileiras e do Distrito Federal.

Apesar da elevada prevalência da HA e dos riscos potenciais e custos elevados, pouco se sabe sobre alguns comportamentos de idosos hipertensos no Brasil, como a atividade física nos seus quatro domínios e seus fatores determinantes. Este estudo objetiva descrever a prática de atividade física em quatro domínios em população de 60 anos ou mais que referiu HA e analisar a prática recomendada de atividade física em cada domínio segundo variáveis demográficas e socioeconômicas.

\section{MÉTODOS}

O Inquérito de Saúde no Município de São Paulo (ISA-Capital) foi um estudo de corte transversal, cujas informações foram coletadas no ano de 2003 no Município de São Paulo. A pesquisa foi realizada por três universidades públicas do Estado (Universidade de São Paulo - USP, Universidade Estadual Paulista - UNESP e Universidade Estadual de Campinas - UNI(AMP) e contou com a parceria da Prefeitura do Município de São Paulo, com financiamento da Fundação de Amparo à Pesquisa do Estado de São Paulo (Projeto de Políticas Públicas, processo no. 88/14099) e da Secretaria Estadual de Saúde de São Paulo. Este estudo foi aprovado pelo Comitê de Ética da Faculdade de Saúde Pública da USP.

A amostragem foi probabilística, por conglomerados, em três estágios:

$1^{\circ}$. estágio: sorteio de setores censitários da PNAD;

$2^{\circ}$. estágio: sorteio de setores censitários do ISA-Capital;

$3^{\circ}$. estágio: sorteio de domicílios.

As unidades primárias de amostragem (setores censitários) foram sorteadas com probabilidade proporcional ao tamanho, a partir dos dados do Censo Populacional do IBGE (Instituto Brasileiro de Geografia e Estatística) de 2000. Os domicílios foram sorteados com probabilidade inversamente proporcional ao tamanho do setor, a partir de listagens feitas em visitas aos setores sorteados.

Para realizar o cálculo do tamanho da amostra, considerou-se a expressão algébrica utilizada na estimação de proporções:

$$
n_{0}=\frac{P(1-P)}{d^{2} / z^{2}} \cdot d e f f
$$

Os valores aplicados a ela foram:

$z=1,96$, valor na curva normal reduzida, correspondente ao nível de confiança de $95 \%$;

$d=0,06$, erro de amostragem admitido;

deff $=1,5$ o efeito do delineamento e;

$P=0,50$, proporção a ser estimada. Tomou-se esse valor para $\mathrm{P}$ por ser o que leva à obtenção de tamanhos de amostras conservadores, uma vez que várias proporções foram estimadas no estudo.

Dessa forma, $n_{\circ}=400$ em cada domínio amostral (idosos do sexo feminino e idosos do sexo masculino). A seguir, fixou-se em sete o número de pessoas a serem entrevistadas em cada unidade primária de amostragem, arredondando para 60 o número de setores a ser sorteado $(400 / 7=57,2)$. Esses valores elevaram a amostra para $420(60 \times 7=420)$.

O Município foi estratificado segundo escolaridade do chefe de família, considerando o percentual de chefes com grau universitário em três níveis: até 5\%; $5 \%$ (inclusive) a 25\%; e $25 \%$ ou mais, conforme dados disponíveis no Censo IBGE de 2000.

O sorteio teve início a partir da listagem dos 264 setores censitários urbanos do Município de São Paulo que fizeram parte da PNAD (Pesquisa Nacional por Amostra de Domicílios) 2002, sendo sorteados 60 setores para amostra dessa pesquisa.

Os dados sofreram ainda nova ponderação no que se refere ao ajuste de pós-estratificação pela escolaridade dos chefes dos domicílios, considerando quatro grupos: com menos de três anos de escolaridade; de quatro a sete anos; de oito a 11 anos; e com 12 ou mais anos de estudo. Essa ponderação visou ajustar a distribuição da amostra à observada no Censo IBGE 2000. Para isso, foram calculadas as razões entre as proporções de pessoas da amostra do ISA-Capital nos novos estratos criados e as observadas no Censo de 2000.

Ao final, dos 872 indivíduos idosos pertencentes à base de dados original do ISA-Capital, a população do presente estudo incluiu os 395 indivíduos com 60 anos ou mais de idade, não-institucionalizados, de ambos os sexos e portadores de HA referida, residentes em área urbana do Município de São Paulo. As informações foram obtidas por meio de questionários aplicados por entrevistadores treinados, respondidos diretamente pelos moradores idosos sorteados. A descrição detalhada do plano amostral do ISA-Capital 2003 já foi objeto de outra publicação. ${ }^{7}$

Os dados sobre atividade física em quatro domínios foram coletados por meio do Questionário Internacional de Atividade Física (IPAQ), forma longa, já validado inclusive para uso em população idosa no Brasil..$^{8,9}$ As questões foram referentes aos tipos de atividades físicas realizadas, a frequência semanal e o tempo total diário nas atividades em cada um dos quatro domínios investigados. Os escores de prática de atividade física em minutos/semana foram calculados segundo as diretrizes internacionais do IPAQ (www.ipaq.ki.se), multiplicando as atividades físicas vigorosas por dois, de acordo com Hallal et al. $2004^{10}$ e Florindo et al. 2009. ${ }^{11}$ A partir dos escores de tempo/semana de prática de AF, optou-se por utilizar o ponto de corte de 150 minutos $/$ semana $^{12}$ em cada domínio. Esta categorização foi escolhida a partir das diretrizes mais recentes para prática de AF em adultos e idosos. ${ }^{11}$ Foram considerados insuficientemente ativos os entrevistados que relataram prática de AF semanal em cada domínio por tempo inferior a 150 minutos.

As variáveis dependentes deste estudo foram catego- 
rizadas segundo pelo menos 150 minutos por semana para cada um dos domínios da AF, ou seja, foram avaliadas quatro variáveis segundo a prática recomendada de AF nos domínios lazer, locomoção/deslocamento, trabalho e doméstica. ${ }^{11}$

As variáveis independentes estudadas foram sexo, faixa etária, situação conjugal, escolaridade e renda familiar mensal em salários-mínimos per capita.

$\mathrm{Na}$ análise geral dos dados, os programas de processamento e análise incorporaram os fatores de ponderação da amostra, baseados no sexo e idade dos entrevistados e na fração amostral do setor censitário, a partir do percentual de chefes de família com nível universitário. Os cálculos para definições dos pesos utilizaram as informações do Censo IBGE de 2000.

Para avaliar o efeito das variáveis demográficas e socioeconômicas sobre a prática recomendada de AF em cada um dos domínios, foi realizada Regressão de Poisson com estimação robusta - modelagem que proporciona melhor ajuste e estima diretamente as razões de prevalências e seus intervalos de confiança. ${ }^{13-15}$

Para a análise de Regressão de Poisson simples, todas as variáveis foram avaliadas em categorias e transformadas em variáveis indicadoras, permitindo a comparação das razões de prevalência entre as categorias de cada variável analisada. As variáveis que obtiveram $p$ menor que 0,10 na análise foram incluídas nos modelos múltiplos. Optamos pela modelagem stepwise forward. As variáveis que permaneceram significativas foram mantidas nos modelos múltiplos finais. Possíveis interações entre as variáveis foram testadas. Tendências lineares foram exploradas para variáveis categóricas ordinais.

Foram utilizados os softwares SPSS (Statistical Package for the Social Sciences), módulo Complex Samples, versão 15.0 e Stata, módulo Survey, versão 9.2. Em todas as análises foi utilizado nível de significância de 5\%.

\section{RESULTADOS}

A amostra total de idosos entrevistados no ISA-Capital 2003 incluiu 872 idosos, sendo que destes, 395 (46,2\%) referiram ser portadores de HA. Dentre os hipertensos referidos, $231(67,6 \%)$ indivíduos pertenciam ao sexo feminino e 164 $(32,4 \%)$ ao sexo masculino. A maioria estava na faixa etária $60-69$ anos $(48,8 \%)$ e vivia com companheiro $(62,4 \%)$. A escolaridade predominante foi de $4-7$ anos $(41,6 \%)$ e $51,9 \%$ dos entrevistados tinham renda familiar mensal inferior a 1,5 salários-mínimos per capita (Tabela 1).

A maior frequência de idosos ativos foi encontrada na prática de AF doméstica (41,4\%). Os domínios lazer e locomo-

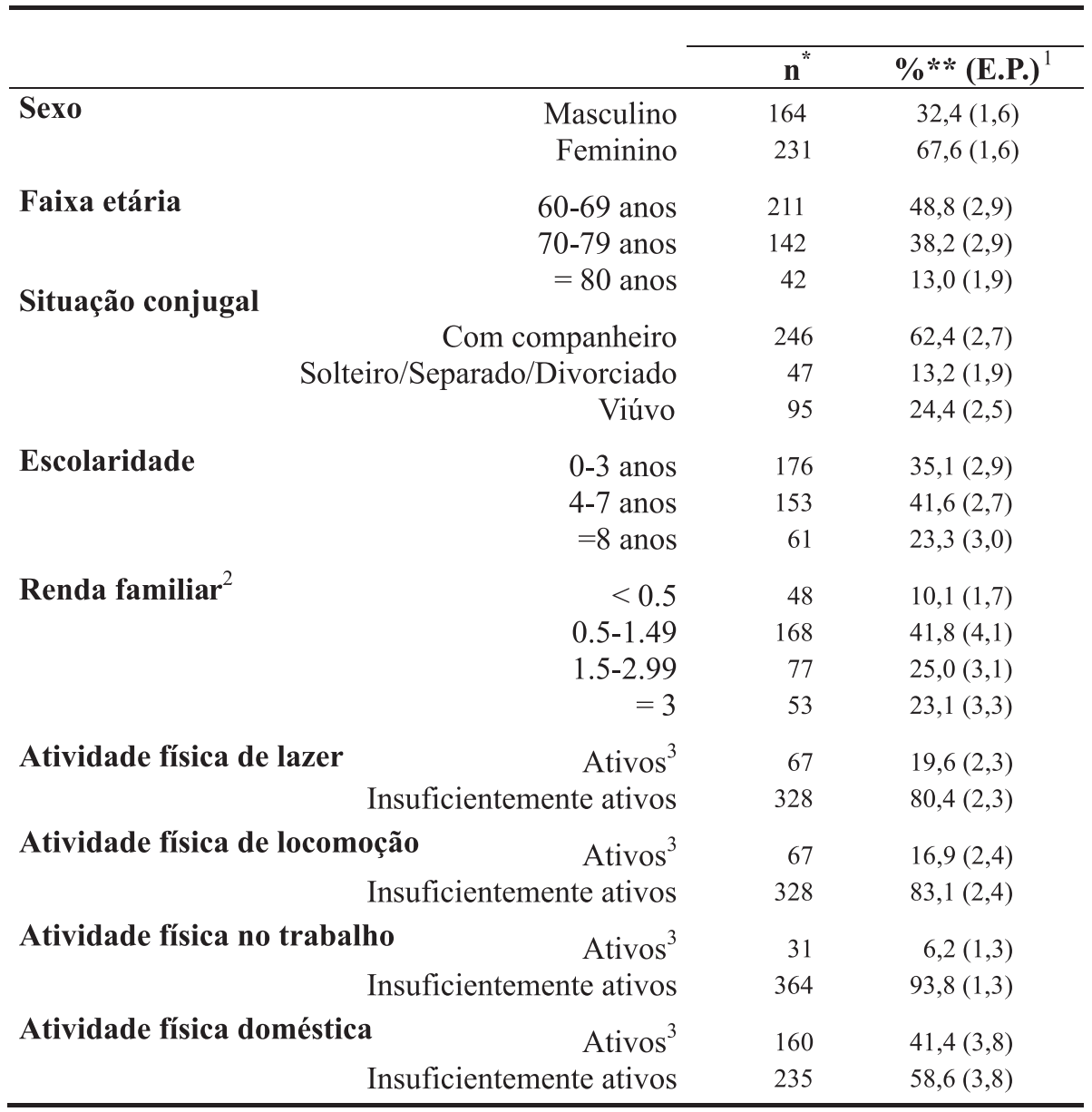

* Números absolutos na amostra não-ponderada. ${ }^{* *}$ Porcentagens na amostra ponderada.

1 Erro-padrão.

2 Renda familiar mensal em salários mínimos per capita.

3 Prática de atividade física $=150$ minutos $/$ semana 
ção apresentaram, respectivamente, 19,6\% e 16,9\% de prática recomendada de AF de 150 minutos por semana. A menor prevalência foi observada no domínio trabalho, com apenas $6,2 \%$ de idosos ativos (Tabela 1 ).

Quanto à prática recomendada de $\mathrm{AF}$ de lazer, observou-se associação direta com a escolaridade ( $p$ tendência $<0,001$ ), sendo que os idosos com maior tempo de estudo foram mais ativos no domínio lazer (Tabela 2). Em relação à $A F$ de locomoção, não houve associações estatisticamente significativas com nenhuma das variáveis sociodemográficas avaliadas neste estudo (Tabela 3 ).

A prática de AF no domínio trabalho associou-se ao sexo masculino $(\mathrm{RP}=3,42$; IC 95\%: 1,75-6,67) e à menor faixa etária ( $p$ tendência $=0,004$ ) (Tabela 4$)$. Quanto à AF doméstica, observou-se menor prática no sexo masculino ( $R P=0,39$; IC 95\%: $0,25-0,61$ ) e associação inversa com a faixa etária ( $p$ tendência $=0,002$ ). Houve ainda maior prática de AF doméstica na população idosa viúva ( $R P=1,51$; IC 95\%: 1,08-2,11), mesmo após ajuste para sexo e faixa etária.

\section{DISCUSSÃO}

O presente estudo demonstrou elevada prevalência de inatividade física na população idosa com hipertensão arterial referida no Município de São Paulo. Nos domínios AF no trabalho e AF doméstica verificou-se diferença entre os gêneros que pode ser justificada segundo os hábitos e aspectos socioculturais aos quais a população idosa está inserida. Foi verificada, também, associação direta da prática recomendada de $\mathrm{AF}$ no lazer com a escolaridade.
A amostra foi constituída por idosos hipertensos referidos, os quais representam uma parcela da população idosa que já teve acesso a serviço de saúde e diagnóstico prévio de HA. Vários estudos, ${ }^{16-18}$ inclusive em população brasileira, ${ }^{19}$ já validaram o uso da hipertensão arterial autorreferida para estudos de base populacional.

Este estudo mostrou associação direta da prática de AF no lazer com a escolaridade. Destaca-se a semelhança com outros estudos que abordam a mesma temática ${ }^{20,21}$ - sendo bastante clara na literatura a maior prática de atividade física no domínio lazer em indivíduos com maior escolaridade. ${ }^{21}$

Em relação à prática recomendada de atividade física no trabalho, foi encontrada importante associação direta com o sexo masculino e associação inversa com a faixa etária, observando-se menor prática de atividade física no trabalho com o aumento da idade. Nesse contexto, pode-se afirmar que os achados acompanham resultado obtido em estudo de Florindo et. al 200911, no qual o sexo feminino apresenta-se menos ativo em todos os domínios da atividade física, excluindo-se o doméstico, e principalmente, menos ativo na atividade física no trabalho.

Tal relação pode ser elucidada, ainda, sob o ponto de vista do contexto histórico cultural, onde homens realizariam trabalhos mais penosos que requereriam maior vigor e desgaste físico fora das residências e por isso continuariam, em idade avançada, a realizar tais atividades, o que vai de acordo com o resultado da análise. ${ }^{22}$

Já os resultados obtidos com a análise da prática de atividade física doméstica evidenciaram que o sexo feminino demográficas e socioeconômicas em população idosa com HA referida, Município de São Paulo.

\begin{tabular}{|c|c|c|c|c|c|}
\hline \multirow{2}{*}{ Variável } & \multirow[b]{2}{*}{$\% 1$} & \multicolumn{2}{|c|}{ Razão de Prevalências } & \multicolumn{2}{|c|}{ Razão de Prevalências } \\
\hline & & Bruta & IC 95\% & Ajustada $^{2}$ & IC $95 \%$ \\
\hline \multicolumn{6}{|l|}{ Sexo } \\
\hline Feminino & 16,2 & 1,00 & & 1,00 & \\
\hline Masculino & 26,7 & 1,64 & $0,98-2,76$ & 1,27 & $0,68-2,39$ \\
\hline \multicolumn{6}{|l|}{ Faixa etária } \\
\hline 60-69 anos & 24,2 & 1,00 & & 1,00 & \\
\hline 70-79 anos & 16,1 & 0,66 & $0,38-1,16$ & 0,71 & $0,37-1,37$ \\
\hline$=80$ anos & 12,8 & 0,52 & $0,21-1,28$ & 0,53 & $0,20-1,40$ \\
\hline \multicolumn{6}{|l|}{ Situação Conjugal } \\
\hline Com companheiro & 24,5 & 1,00 & & 1,00 & \\
\hline Solteiro/Divorciado & 6,8 & 0,27 & $0,08-0,85$ & 0,47 & $0,16-1,42$ \\
\hline \multirow{2}{*}{ Viúvo } & 15,1 & 0,61 & $0,36-1,04$ & 0,91 & $0,55-1,51$ \\
\hline & & Teste de tendência & $=0,027$ & & \\
\hline \multicolumn{6}{|l|}{ Escolaridade } \\
\hline 0-3 anos & 9,2 & 1,00 & & 1,00 & \\
\hline 4-7 anos & 23,3 & 2,34 & $1,33-4,10$ & 2,29 & $1,25-4,19$ \\
\hline$=8$ anos & 28,2 & 2,83 & $1,49-5,38$ & 2,16 & $0,96-4,84$ \\
\hline \multicolumn{6}{|l|}{ Renda Familiar ${ }^{3}$} \\
\hline$<0.5$ & 12,7 & 1,00 & & 1,00 & \\
\hline $0.5-1.49$ & 17,6 & 1,27 & $0,42-3,88$ & 0,96 & $0,37-2,44$ \\
\hline $1.5-2.99$ & 25,5 & 1,85 & $0,62-5,50$ & 1,08 & $0,40-2,93$ \\
\hline$=3$ & 22,3 & 1,61 & $0,54-4,79$ & 0,92 & $0,33-2,56$ \\
\hline
\end{tabular}

Teste de tendência

1 Porcentagens na amostra ponderada.

2 Regressão de Poisson ajustada para variáveis apresentadas na tabela

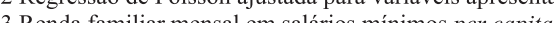




\begin{tabular}{|c|c|c|c|c|c|}
\hline \multirow{2}{*}{ Variável } & \multirow[b]{2}{*}{$\% 1$} & \multicolumn{2}{|c|}{ Razão de Prevalências } & \multicolumn{2}{|c|}{ Razão de Prevalências } \\
\hline & & Bruta & IC $95 \%$ & Ajustada $^{2}$ & IC $95 \%$ \\
\hline \multicolumn{6}{|l|}{ Sexo } \\
\hline Feminino & 14,6 & 1,00 & & 1,00 & \\
\hline Masculino & 21,8 & 1,49 & $0,95-2,35$ & 1,35 & $0,74-2,47$ \\
\hline \multicolumn{6}{|l|}{ Faixa etária } \\
\hline 60-69 anos & 18,1 & 1,00 & & 1,00 & \\
\hline 70-79 anos & 20,4 & 1,12 & $0,65-1,94$ & 1,07 & $0,61-1,86$ \\
\hline$=80$ anos & 2,2 & 0,12 & $0,01-0,94$ & 0,15 & $0,02-1,13$ \\
\hline & & Teste de tendência & $=0,044$ & & \\
\hline \multicolumn{6}{|l|}{ Situação Conjugal } \\
\hline Com companheiro & 18,3 & 1,00 & & 1,00 & \\
\hline Solteiro/Divorciado & 6,1 & 0,33 & $0,12-0,85$ & 0,55 & $0,20-1,47$ \\
\hline Viúvo & 20,3 & 1,10 & $0,64-1,90$ & 1,47 & $0,81-3,91$ \\
\hline & & Teste de tendência & $=0,023$ & & \\
\hline \multicolumn{6}{|l|}{ Escolaridade } \\
\hline 0-3 anos & 12,2 & 1,00 & & 1,00 & \\
\hline 4-7 anos & 18,4 & 1,42 & $0,74-2,74$ & 1,48 & $0,71-3,08$ \\
\hline$=8$ anos & 20,7 & 1,61 & $0,76-3,40$ & 1,78 & $0,81-3,91$ \\
\hline \multicolumn{6}{|l|}{ Renda Familiar ${ }^{3}$} \\
\hline$<0.5$ & 10,0 & 1,00 & & 1,00 & \\
\hline $0.5-1.49$ & 17,6 & 1,60 & $0,65-3,92$ & 1,26 & $0,52-3,06$ \\
\hline $1.5-2.99$ & 19,2 & 1,73 & $0,70-4,23$ & 1,18 & $0,47-2,96$ \\
\hline$=3$ & 17,3 & 1,55 & $0,60-4,00$ & 1,02 & $0,37-2,80$ \\
\hline
\end{tabular}

\section{Tabela 4}

Prática recomendada ( $\geq 150 \mathrm{~min} /$ semana) de atividade física no trabalho (\%) segundo variáveis demográficas e socioeconômicas em população idosa com HA referida, Município de São Paulo.

\begin{tabular}{|c|c|c|c|c|c|}
\hline \multirow[b]{2}{*}{ Variável } & \multirow[b]{2}{*}{$\%{ }^{1}$} & \multicolumn{2}{|c|}{ Razão de Prevalências } & \multicolumn{2}{|c|}{ Razão de Prevalências } \\
\hline & & Bruta & IC 95\% & Ajustada $^{2}$ & IC $95 \%$ \\
\hline \multicolumn{6}{|l|}{ Sexo } \\
\hline Feminino & 3,0 & 1,00 & & 1,00 & \\
\hline Masculino & 12,9 & 4,32 & 2,13-8,77 & 3,42 & $1,75-6,67$ \\
\hline & & Teste de tendência & $=\mathbf{0 , 0 0 0}$ & Teste de tendência & $=\mathbf{0 , 0 0 0}$ \\
\hline \multicolumn{6}{|l|}{ Faixa etária } \\
\hline 60-69 anos & 11,6 & 1,00 & & 1,00 & \\
\hline $70-79$ anos & 1,4 & 0,12 & $0,03-0,46$ & 0,15 & $0,04-0,61$ \\
\hline$=80$ anos & 0 & - & & - & \\
\hline & & Teste de tendência & $=0,001$ & Teste de tendência & $=0,004$ \\
\hline \multicolumn{6}{|l|}{ Situação Conjugal } \\
\hline Com companheiro & 3,9 & 1,00 & & 1,00 & \\
\hline Solteiro/Divorciado & 5,5 & 0,79 & $0,29-2,19$ & 1,12 & $0,30-4,08$ \\
\hline Viúvo & 4,2 & 0,60 & $0,20-1,81$ & 1,28 & $0,35-4,63$ \\
\hline \multicolumn{6}{|l|}{ Escolaridade } \\
\hline 0-3 anos & 6,3 & 1,00 & & 1,00 & \\
\hline 4-7 anos & 6,0 & 0,98 & $0,41-2,33$ & 0,57 & $0,20-1,60$ \\
\hline$=8$ anos & 6,8 & 1,11 & $0,40-3,08$ & 0,70 & $0,22-2,23$ \\
\hline \multicolumn{6}{|l|}{ Renda Familiar ${ }^{3}$} \\
\hline$<0.5$ & 4,4 & 1,00 & & 1,00 & \\
\hline $0.5-1.49$ & 5,1 & 1,16 & $0,28-4,70$ & 1,24 & $0,39-3,87$ \\
\hline $1.5-2.99$ & 10,4 & 2,38 & $0,45-12,60$ & 2,00 & $0,46-8,70$ \\
\hline$=3$ & 4,5 & 1,04 & $0,17-6,36$ & 1,03 & $0,16-6,73$ \\
\hline
\end{tabular}




\begin{tabular}{|c|c|c|c|c|c|}
\hline \multirow[b]{2}{*}{ Variável } & \multirow[b]{2}{*}{$\%^{1}$} & \multicolumn{2}{|c|}{ Razão de Prevalências } & \multicolumn{2}{|c|}{ Razão de Prevalências } \\
\hline & & Bruta & IC $95 \%$ & Ajustada $^{2}$ & IC $95 \%$ \\
\hline \multicolumn{6}{|l|}{ Sexo } \\
\hline Feminino & 52,4 & 1,00 & & 1,00 & \\
\hline Masculino & 18,4 & 0,35 & $0,24-0,51$ & 0,39 & $0,25-0,61$ \\
\hline & & Teste de tendência & $=0,000$ & Teste de tendência & $=0,000$ \\
\hline \multicolumn{6}{|l|}{ Faixa etária } \\
\hline 60-69 anos & 48,3 & 1,00 & & 1,00 & \\
\hline $70-79$ anos & 37,6 & 0,77 & $0,58-1,03$ & 0,71 & $0,52-0,95$ \\
\hline$=80$ anos & 26,6 & 0,55 & $0,30-1,00$ & 0,50 & $0,30-0,83$ \\
\hline \multicolumn{6}{|l|}{ Situação Conjugal } \\
\hline Com companheiro & 32,2 & 1,00 & & 1,00 & \\
\hline Solteiro/Divorciado & 49,8 & 1,54 & $1,03-2,31$ & 1,21 & $0,80-1,82$ \\
\hline Viúvo & 60,9 & 1,89 & $1,35-2,63$ & 1,51 & $1,08-2,11$ \\
\hline \multirow{2}{*}{\multicolumn{6}{|c|}{ Escolaridade }} \\
\hline & & & & & \\
\hline 0-3 anos & 41,2 & 1,00 & & 1,00 & \\
\hline 4-7 anos & 53,5 & 1,29 & $0,97-1,71$ & 1,11 & $0,84-1,48$ \\
\hline$=8$ anos & 19,8 & 0,47 & $0,27-0,82$ & 0,65 & $0,35-1,20$ \\
\hline \multicolumn{6}{|l|}{ Renda Familiar ${ }^{3}$} \\
\hline$<0.5$ & 33,6 & 1,00 & & 1,00 & \\
\hline $0.5-1.49$ & 47,1 & 1,36 & $0,79-2,34$ & 1,36 & $0,82-2,26$ \\
\hline $1.5-2.99$ & 47,8 & 1,38 & $0,78-2,45$ & 1,51 & $0,90-2,53$ \\
\hline$=3$ & 34,1 & 0,99 & $0,53-1,82$ & 1,21 & $0,70-2,09$ \\
\hline
\end{tabular}

$(p<0,001)$, menor faixa etária $(p=0,002)$ e a situação conjugal de viuvez $(p=0,017)$ tiveram associação positiva com a prática dos mínimos 150 minutos semanais desta atividade.

Entre outras razões, no que se refere ao sexo feminino e a prática de maior atividade física doméstica, está o fator cultural. ${ }^{22}$ Resultados semelhantes foram encontrados em outros estudos, tanto no Brasil, ${ }^{11}$ quanto em outros países, como a Inglaterra ${ }^{23}$ e a Austrália. ${ }^{24}$ Embora, pesquisas recentes coloquem em dúvida os benefícios no controle da hipertensão arterial advindos da prática de atividade física no domínio doméstico. ${ }^{25}$

Os resultados obtidos a partir das análises da atividade física de locomoção não apresentaram valores de associação significativos em relação às variáveis estudadas. Entretanto, a prevalência de prática de atividade física de locomoção foi baixa em toda a população avaliada e deve ser estimulada como importante ferramenta auxiliar na manutenção de um estilo de vida ativo.

Este estudo apresenta pontos a serem considerados, como o caráter transversal da coleta de dados que traz um retrato de determinado espaço tempo da população, a escassez de estudos nacionais com temas semelhantes que possibilitem o confrontamento dos achados, e o fato do instrumento de coleta - IPAQ longo - não ser amplamente aceito, apesar de sua validação. Outro fator importante a ser considerado no estudo em questão é a redução do $n$ inicial que pode influenciar no poder estatístico dos testes utilizados.

É importante ressaltar a contribuição de trabalhos semelhantes a este para a reflexão de temas como a prática de atividade física, promoção da saúde, tratamento e controle de doenças crônicas, que se tornam cada vez mais preocupantes para o setor público de saúde, assim como a crescente prevalência hipertensão arterial e o envelhecimento populacional, vivenciado na atualidade. O caráter representativo populacional da pesquisa, do município de São Paulo, relacionado à avaliação da atividade física, abordando as diferentes características dos quatro domínios, e o método de coleta direta dos dados, através de um questionário aplicado segundo uma lógica amostral gerada para contemplar a população em sua heterogeneidade, são pontos fortes a serem considerados.

Em conclusão, este estudo serve, entre outras contribuições, para a caracterização da atividade física na população idosa acometida por hipertensão arterial sistêmica, doença crônica de maior prevalência mundial. Aspecto que auxilia na compreensão dos hábitos desta população e contribui para fomentar discussões a respeito de programas de controle, manutenção, promoção de saúde e qualidade de vida, não só através da prática de atividade física como único recurso, mas como forma de combate aos altos índices de morbidade e mortalidade gerados pela hipertensão arterial e seus agravos, bem como os gastos do sistema público de saúde. Outra importante contribuição são os indicadores e dados que demonstram os índices de atividade/sedentarismo ${ }^{26}$ populacional, destacados nos quatro domínios existentes da atividade física em uma faixa etária que desperta cada vez maior atenção e preocupação, pensando em um futuro de transição acentuada da pirâmide demográfica no Brasil.

\section{REFERÊNCIAS}

1. Haskell W, Lee I, Pate R, et al. Physical activity and public health: updated recommendation for adults from the American College of Sports Medicine and the American Heart Association. Med Sci Sports Exerc 2007;39:1423-1434. 
2. World Health Organization. Global strategy on diet, physical activity and health. 2004. Available in: www.who.int.hpr.

3. Nelson M, Rejeski W, Blair S, et al. Physical activity and public health in older adults: recommendation from the American College of Sports Medicine and the American Heart Association. Circulation 2007;116:1094-1105.

4. Kearney P, Whelton M, Reynolds K, et al. Global burden of hypertension: analysis of worldwide data. Lancet 2005;365:217-223.

5. Ferreira S, Moura E, Malta D, Sarno F. Frequency of arterial hypertension and associated factors: Brazil, 2006. Rev Saude Publica 2009;43 Suppl 2:98-106.

6. Lima e Costa M, Peixoto S, César C, Malta D, Moura E. Health behaviors among older adults with hypertension, Brazil, 2006. Rev Saude Publica 2009;43 Suppl 2:18-26.

7. Alves M. ISA-Capital 2003: Plano de Amostragem. São Paulo: 2003. Available in: http://www.fsp.usp.br/isa-sp/pdf/planoamostralisacapital.pdf.

8. Benedetti T, Antunes P, Rodriguez-Anez C, Mazo G, Petroski E. Reprodutibilidade e validade do Questionário Internacional de Atividade Física (IPAQ) em homens idosos. Rev Bras Med Esporte 2007;13:11-16.

9. Craig $C$, Marshall $A$, Sjöström $M$, et al. International physical activity questionnaire: 12-country reliability and validity. Med Sci Sports Exerc 2003;35:13811395.

10. Hallal P, Victora C. Reliability and validity of the International Physical Activity Questionnaire (IPAQ). Med Sci Sports Exerc 2004;36:556.

11. Florindo A, Guimarães V, Cesar C, et al. Epidemiology of leisure, transportation, occupational, and household physical activity: prevalence and associated factors. J Phys Act Health 2009;6:625-632.

12. U.S. Department of Health and Human Services. 2008 Physical activity guidelines for Americans. Washington DC: USDHHS; 2008.

13. Skov T, Deddens J, Petersen M, Endahl L. Prevalence proportion ratios: estimation and hypothesis testing. Int J Epidemiol 1998;27:91-95.

14. Barros A, Hirakata V. Alternatives for logistic regression in cross-sectional studies: an empirical comparison of models that directly estimate the prevalence ratio. BMC Med Res Methodol 2003;3:21.

15. Francisco $P$, Donalisio $M$, Barros $M$, et al. Association measures in cross-sectio- nal studies with complex samplings: odds ratio and prevalence ratio. Rev Bras Epidemiol 2008;11:347-355.

16. Giles W, Croft J, Keenan N, Lane M, Wheeler F. The validity of self-reported hypertension and correlates of hypertension awareness among blacks and whites within the stroke belt. Am J Prev Med 1995;11:163-169.

17. Vargas C, Burt V, Gillum R, Pamuk E. Validity of self-reported hypertension in the National Health and Nutrition Examination Survey III, 1988-1991. Prev Med 1997;26:678-685.

18. Wang $Y$, Jiang $Y$, Zhang M. [Validity of self-reported hypertension among Chinese]. Zhonghua Liu Xing Bing Xue Za Zhi 2009;30:414-416.

19. Lima-Costa M, Peixoto S, Firmo J. [Validity of self-reported hypertension and its determinants (the Bambuí study)]. Rev Saude Publica 2004;38:637-642.

20. Zaitune MPA, et al. Fatores associados ao sedentarismo no lazer em idosos, Campinas, São Paulo, Brasil. Cad. Saúde Pública, Rio de Janeiro, 2007 23(6):1329-1338.

21. Dias-da-Costa JS, et al. Epidemiology of leisure-time physical activity: a population-based study in southern Brazil. Cad. Saúde Pública, Rio de Janeiro, 2005 21(1):275-282.

22. Jurakić D et al. Physical Activity of Croatian Population: Cross-sectional Study Using International Physical Activity Questionnaire. Croat Med J. 2009; 50 165-73.

23. Lawlor DA, Taylor M, Bedford C, et al. Is housework good for health? Levels of physical activity and factors associated with activity in elderly women. Results from the British Women's Heart and Health Study. J Epidemiol Community Health 2002;56:473- 8

24. Phongsavan P, Merom D, Marshall AL, et al. Estimating physical activity levels: the role of domestic activities. J Epidemiol Community Health 2004;58:466 -7

25. Stamatakis E et al. Domestic Physical Activity in Relationship to Multiple CVD Risk Factors. Am J Prev Med 2007;32(4).

26. Hughes JP. Leisure-Time Physical Activity Among US Adults 60 or More Years of Age: Results From NHANES 1999-2004. Journal of Physical Activity and Health $2008,5,347-358$ 